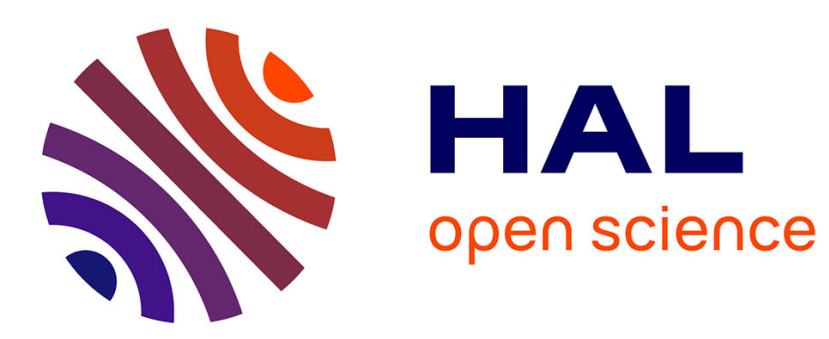

\title{
Robust optimal control of stochastic hyperelastic materials
}

\author{
J Martínez-Frutos, Rogelio Ortigosa, P Pedregal, F Periago
}

\section{To cite this version:}

J Martínez-Frutos, Rogelio Ortigosa, P Pedregal, F Periago. Robust optimal control of stochastic hyperelastic materials. Applied Mathematical Modelling, 2020. hal-02440704

\section{HAL Id: hal-02440704 https://hal.science/hal-02440704}

Submitted on 15 Jan 2020

HAL is a multi-disciplinary open access archive for the deposit and dissemination of scientific research documents, whether they are published or not. The documents may come from teaching and research institutions in France or abroad, or from public or private research centers.
L'archive ouverte pluridisciplinaire HAL, est destinée au dépôt et à la diffusion de documents scientifiques de niveau recherche, publiés ou non, émanant des établissements d'enseignement et de recherche français ou étrangers, des laboratoires publics ou privés. 


\title{
Robust optimal control of stochastic hyperelastic materials
}

\author{
J. Martínez-Frutos ${ }^{\dagger 1}$, R. Ortigosa ${ }^{\dagger}$, P. Pedregal ${ }^{\ddagger}$, F. Periago ${ }^{\dagger}$ \\ $\dagger$ Computational Mechanics and Scientific Computing Group, \\ Technical University of Cartagena, Campus Muralla del Mar, 30202, Cartagena (Murcia), Spain \\ $\ddagger I N E I$, Universidad de Castilla-La Mancha, 13071 Ciudad Real, Spain
}

\begin{abstract}
Soft robots are highly nonlinear systems made of deformable materials such as elastomers, fluids and other soft matter, that often exhibit intrinsic uncertainty in their elastic responses under large strains due to microstructural inhomogeneity. These sources of uncertainty might cause a change in the dynamics of the system leading to a significant degree of complexity in its controllability. This issue poses theoretical and numerical challenges in the emerging field of optimal control of stochastic hyperelasticity. This paper states and solves the robust averaged control in stochastic hyperelasticity where the underlying state system corresponds to the minimization of a stochastic polyconvex strain energy function. Two bio-inspired optimal control problems under material uncertainty are addressed. The expected value of the $L^{2}$-norm to a given target configuration is minimized to reduce the sensitivity of the spatial configuration to variations in the material parameters. The existence of optimal solutions for the robust averaged control problem is proved. Then the problem is solved numerically by using a gradientbased method. Two numerical experiments illustrate both the performance of the proposed method to ensure the robustness of the system and the significant differences that may occur when uncertainty is incorporated in this type of control problems.
\end{abstract}

Keywords: Robust optimal control , hyperelasticity, material uncertainty, turgor pressure, active fibers, soft robotics.

\section{Introduction}

Since the early 1940s, the field of soft robotics continues exploring the creation of machines with biomimetic dexterous features superseding the capabilities of humans. This field represents a radically different paradigm with respect to the conventional field of hard robotics. The latter, which enabled an outstanding technological revolution in industrial automation, autonomous vehicles, etc. perform extraordinarily well for the specific tasks that they have been purposely designed for. The question that emerges then is therefore evident: if conventional robotics perform exceptionally, why to pursue a completely opposite robotic paradigm? Not to mention

${ }^{1}$ Corresponding author: jesus.martinez@upct.es 
that soft robotics are made out of highly deformable materials such as elastomers, fluids and other soft matter, entailing a much higher degree of complexity in their controllability. The answer to this question lies on the programability of soft robotics for a wider range of tasks than their hard counterparts, along with their adaptability to rapidly changing uncertain conditions while performing these tasks [1].

As already mentioned, the controllability of soft robots, potentially actuated by means of a wide spectrum of complex external stimuli (electric or magnetic field, mechanical pressure, osmotic pressure, etc.) is not a trivial task. This complexity reflects also in the numerical modelling of these soft materials, specially when aleatory sources of uncertainty such as random material properties, random geometry or random environmental conditions, are incorporated in the numerical model. This fact, together with the development of probabilistic uncertainty propagation methods, has fostered the interest in models represented by Partial Differential Equations (PDEs) with random inputs [2, 3, 4]. In the context of stochastic hyperelasticity, large random variabilities observed in the bulk and shear moduli of Ogden-type hyperelastic materials, typically used to model elastomeric materials and soft biological tissues, are reported by recent works in [5, 6, 7]. Stochastic versions of the polyconvex energy functionals of these constitutive models have been proposed and validated against experimental data in those references.

The literature on optimal control of hyperelastic materials adopting a rigorous mathematical prism is relatively scarce even in the deterministic setting [8, 9]. In these works it is wellaccepted to consider polyconvex strain energy functionals [10, 11, 12] defining the constitutive model of the elastomer or soft material. Ball proves in his seminal paper [10] that polyconvexity and coercivity (i.e. growth conditions) of the strain energy density entails the existence of minimisers of the total energy of the elastomer. Examples of isotropic polyconvex strain energy functions are the Odgen model, the Neo-Hookean model, the Mooney-Rivlin model, etc. The deterministic assumption made in most works concerning optimal control of highly deformable materials might be very convenient from the analysis and numerical standpoints. However, soft materials often exhibit intrinsic variability in their elastic responses under large strains leading to a significant degree of complexity in its controllability. Thus, accuracy and realism with respect to the physical response of a deformable soft material requires the incorporation of relevant sources of uncertainty in the numerical model. In this sense, a framework, based on information theory, for the modeling of stochastic Ogden class of stored energy functions is presented in [5, 6, 7]. The proposed models are almost surely consistent with the theory of nonlinear elasticity and are able to mimic the mean behavior and variability that are typically encountered in the experimental characterization of biological tissues.

The problem of optimal control of Stochastic PDEs has sparked significant interest last years. Existence results and, computationally efficient numerical resolution methods have been developed during the last two decades, but only for the case of random linear PDEs. In particular, in [13] the concept of averaged controllability was introduced and positive averaged controllability results have been recently obtained for parameter dependent evolution PDEs 
[14, 15]. Although controlling the average of the state variable provides a first idea of robustness, it may be useless in scenarios where the dispersion of the system response is large. In the context of optimal control theory, the problem of controlling both the average and the variance of the state variable has been studied in [16, 17, 18] at the theoretical and numerical points of view. Significant differences between optimal controls minimizing only the average of the state variable and controls minimizing both the mean and the variance of the state variable were numerically observed in those references.

To the best of the author's knowledge, the optimal control of soft materials governed by stochastic hyperelastic constitutive models still remains an open issue. This paper addresses, both from the numerical and analysis standpoints, the robust optimal control of materials governed by stochastic hyperelastic constitutive models. A probabilistic framework is used to characterized the random parameters of a stochastic Ogden type stored energy function. Inspired by [8], we consider two bio-inspired types of external actions (denoted as control in the sequel) on the elastomeric material, based on Turgor pressure and active fibers. As it will be shown in this paper, the first resembles the Turgor-based motion in the plant kingdom, whilst the second type of control simulates the electro-activation mechanism of muscles (e.g. the human heart). To take into account the variability of the deformed configuration with respect to desired target configuration, the deterministic cost functionals are averaged with respect to the underlying probability distributions.

The layout of the paper is as follows: Section 2 introduces the notions of hyperelasticity in the stochastic framework. In addition, it is shown how the two types of controls considered can be additively incorporated in the stochastic stored energy function of the material. Section 3 presents the optimal control problem in both deterministic and stochastic frameworks, showing the assumptions under which both control problems (associated with each type of control) are well-posed. Then, existence of optimal solutions for both problems is proved in Section 3.2 . Section 4 describes the proposed, gradient-based, numerical resolution method and analyses two numerical experiments that show the importance of considering the uncertainty of the material parameters in the optimal control problem. Finally, Section 5 provides some concluding remarks and open problems.

\section{Stochastic nonlinear continuum mechanics}

\subsection{Stochastic kinematics}

Let $\mathcal{D}_{0} \subset \mathbb{R}^{N}, N=2,3$, be an open, bounded and connected domain which represents the reference (or undeformed) configuration of an elastic body. Let $(\Omega, \mathcal{F}, \mathbb{P})$ be a complete probability space, where $\Omega$ is the set of outcomes, $\mathcal{F} \subset 2^{\Omega}$ is the $\sigma$-algebra of events, and $\mathbb{P}: \mathcal{F} \rightarrow[0,1]$ is a probability measure. The random deformation of the body $\mathcal{D}_{0}$ is defined through the mapping $\boldsymbol{\Phi}: \mathcal{D}_{0} \times \Omega \rightarrow \mathbb{R}^{N}$, which, for every random even $\omega \in \Omega$, is assumed to be sufficiently smooth, injective and orientation preserving with respect to $\boldsymbol{X} \in \mathcal{D}_{0}$. The mapping

$\boldsymbol{\Phi}$ links a material particle $\boldsymbol{X} \in \mathcal{D}_{0}$ to a particle $\boldsymbol{x} \in \mathcal{D}(\omega) \subset \mathbb{R}^{N}$, the random deformed 
configuration, according to $\boldsymbol{x}=\boldsymbol{\Phi}(\boldsymbol{X}, \omega)$ (see Figure 1). As usual, $\boldsymbol{u}:=\boldsymbol{x}-\boldsymbol{X}$ denotes the displacement field.

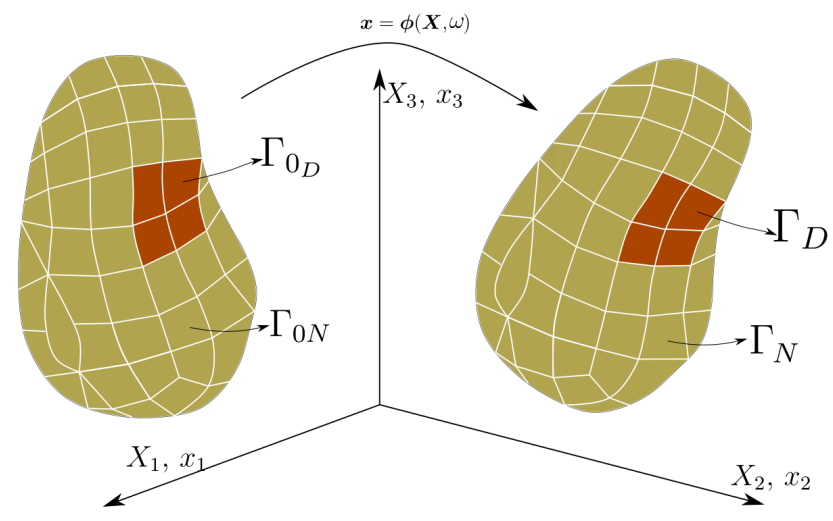

Figure 1: Graphical illustration of the random deformation mapping $\boldsymbol{\Phi}(\cdot, \omega): \mathcal{D}_{0} \rightarrow \mathcal{D}(\omega)$ between reference $\mathcal{D}_{0}$ and random deformed $\mathcal{D}(\omega)$ configurations.

Associated with the mapping $\boldsymbol{\Phi}$, the random deformation tensor $\boldsymbol{F}$ is defined as

$$
\boldsymbol{F}: \mathcal{D}_{0} \times \Omega \rightarrow \mathbb{R}^{N \times N}, \quad \boldsymbol{F}=\nabla_{0} \boldsymbol{\Phi}(\boldsymbol{X}, \omega),
$$

where $\boldsymbol{\nabla}_{0}(\bullet)$ is the material gradient operator with respect to $\boldsymbol{X} \in \mathcal{D}_{0}$. Associated with $\boldsymbol{F}$, its co-factor $\boldsymbol{H}$ and its Jacobian $J$ are defined as

$$
\boldsymbol{H}=(\operatorname{det} \boldsymbol{F})(\boldsymbol{F})^{-T} ; \quad J=\operatorname{det} \boldsymbol{F} .
$$

The orientation-preserving condition is written as $J(\boldsymbol{X}, \omega)>0$ a.e. $\boldsymbol{X} \in \mathcal{D}_{0}$, and for every $\omega \in \Omega$. We assume that the boundary of $\mathcal{D}_{0}$ is smooth and it is decomposed into two (also smooth) disjoint parts: $\Gamma_{0_{D}}$ and $\Gamma_{0_{N}}$. On the Dirichlet boundary $\Gamma_{0_{D}}$, it is imposed $\boldsymbol{\Phi}=\boldsymbol{\Phi}_{0}$ for a given deformation $\Phi_{0}: \mathcal{D}_{0} \rightarrow \mathbb{R}^{N}$, while on $\Gamma_{0_{N}}$, we impose a stress-free boundary condition. Notice that the above boundary conditions do not depend on $\omega \in \Omega$.

\subsection{Stochastic polyconvex constitutive models}

In this paper, we are interested in stochastic (also named random) versions of the classical deterministic polyconvex stored energy densities considerered in hyperelasticity theory. For the sake of clarity in the exposition, in the sequel, we focuss on the following compressible random Mooney-Rivlin model

$$
\begin{aligned}
W(\omega, \boldsymbol{F})=\mathbb{W}(\omega, \boldsymbol{F}, \boldsymbol{H}, J) & =\mu_{1}(\omega)\left(\|\boldsymbol{F}\|^{2}-3\right)+\mu_{2}(\omega)\left(\|\boldsymbol{H}\|^{2}-3\right) \\
& -2\left(\mu_{1}(\omega)+2 \mu_{2}(\omega)\right) \log J+\frac{\mu_{3}(\omega)}{2}(J-1)^{2},
\end{aligned}
$$

where the main novelty here is that the material parameters $\left\{\mu_{1}(\omega), \mu_{2}(\omega), \mu_{3}(\omega)\right\}$ are assumed to be random variables supported in $\mathbb{R}_{*}^{+}:=\{x \in \mathbb{R}: x>0\}$. As a consequence of the positivity 
of these material parameters, the density $W(\omega, \boldsymbol{F})$ in 2.2 satisfies, for each fixed $\omega \in \Omega$, the following three crucial conditions (see [11, Th. 4.10-2]):

Polyconvexity: for the set $\mathcal{V}=\{\boldsymbol{A}, \boldsymbol{B}, c\}$ with $\boldsymbol{A} \in \mathbb{R}^{N \times N}, \boldsymbol{B} \in \mathbb{R}^{N \times N}$ and $c \in \mathbb{R}_{*}^{+}$, the following condition is satisfied

$$
\mathbb{W}\left(\omega, \lambda \mathcal{V}_{1}+(1-\lambda) \mathcal{V}_{2}\right) \leq \lambda \mathbb{W}\left(\omega, \mathcal{V}_{1}\right)+(1-\lambda) \mathbb{W}\left(\omega, \mathcal{V}_{2}\right)
$$

$\forall \lambda \in[0,1]$ and $\forall \mathcal{V}_{1}, \mathcal{V}_{2} \in \mathcal{V}$

\section{Coerciveness:}

$$
\mathbb{W}(\omega, \boldsymbol{F}, \boldsymbol{H}, J) \geq c_{0}(\omega)\left(\|\boldsymbol{F}\|^{2}+\|\boldsymbol{H}\|^{2}+J^{2}\right)+c_{1}(\omega) ; \quad c_{0}(\omega)>0, c_{1}(\omega) \in \mathbb{R} .
$$

Limit behavior 2:

$$
\lim _{J \rightarrow 0^{+}} \mathbb{W}(\omega, \boldsymbol{F}, \boldsymbol{H}, J)=+\infty
$$

As in the deterministic situation, consistency with linearised elasticity in the stochastic framework entails the following relations between the above random material parameters and the shear $\mu(\omega)$ and bulk $\kappa(\omega)$ moduli

$$
\mu_{1}(\omega)+\mu_{2}(\omega)=2 \mu(\omega) ; \quad 4 \mu_{2}(\omega)+\mu_{3}(\omega)=\kappa(\omega)-\frac{2}{3} \mu(\omega) .
$$

Making use of the Maximum Entropy Principle, the authors in [5, 6] show that the random material parameters $\left\{\mu_{1}(\omega), \mu_{2}(\omega), \mu_{3}(\omega)\right\}$ can then be given by

$$
\mu_{1}(\omega)=\frac{\mu(\omega) U}{2} ; \quad \mu_{2}(\omega)=\frac{\mu(\omega)(1-U)}{2} ; \quad \mu_{3}(\omega)=\mu(\omega)\left(2 U-\frac{8}{3}\right)+\kappa(\omega),
$$

where $\{\mu(\omega), \kappa(\omega)\}$ are statistically independent random variables of Gamma-type, and $U$ is a suitable Beta distributed random variable.

\subsection{Bio-inspired controls}

Fostered by [8], two types of bio-inspired controls are considered in the sequel. The effect of both controls can be modeled mathematically by additively decomposing the total stochastic stored energy density, denoted as $W_{\text {tot }}$, as

$$
W_{\mathrm{tot}}(\omega, \boldsymbol{F}, m)=W(\omega, \boldsymbol{F})+W_{m}(\boldsymbol{X}, \boldsymbol{F}) ; \quad W_{m}(m, \boldsymbol{F})=m(\boldsymbol{X}) \hat{W}_{m}(\boldsymbol{F}),
$$

where the first term on the right-hand side of (2.8) corresponds to the purely mechanical contribution $W$ in $(2.2)$, and the second one, namely $W_{m}$, represents the energy induced by the control $m(\boldsymbol{X}): \mathcal{D}_{0} \rightarrow \mathbb{R}$. Notice that there exists an implicit dependence of $W_{m}$ with respect to $\omega \in \Omega$ through the deformation gradient tensor $\boldsymbol{F}=\boldsymbol{F}(\boldsymbol{X}, \omega)$. The explicit expression of $\hat{W}_{m}(\boldsymbol{F})$ for the two types of bio-inspired controls is presented next.

\footnotetext{
${ }^{2}$ The underlying justification for this condition is that it enforces the orientation-preserving condition, namely $J(\boldsymbol{X}, \omega)>0$ a.e $\boldsymbol{X} \in \mathcal{D}_{0}$, and hence the local invertibility and local injectivity of the mapping $\boldsymbol{\Phi}$.
} 

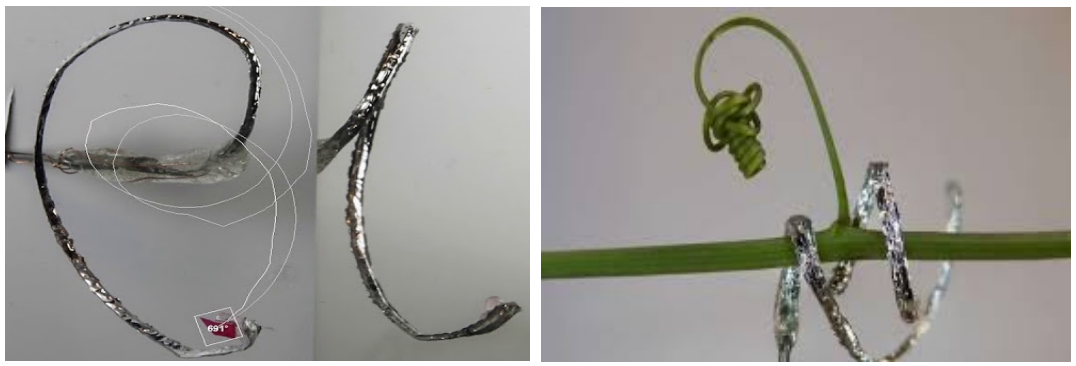

(a)

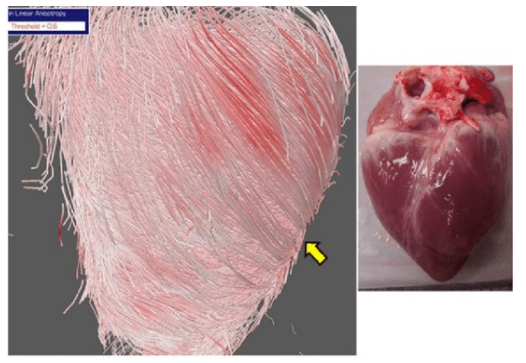

(b)

Figure 2: (a) Tendril-like soft robot based on an osmotic actuation 19. (b) Fiber structure of heart (courtesy of "The Correlation of 3D DT-MRI Fiber Disruption with Structural and Mechanical Degeneration in Porcine Myocardium")

\subsubsection{Turgor-pressure type control}

In this case, the control $m(\boldsymbol{X}): \mathcal{D}_{0} \rightarrow \mathbb{R}$ represents the Turgor pressure and the coupled component $\hat{W}_{m}$ adopts the following expression

$$
\hat{W}_{m}(\boldsymbol{F})=-J(\boldsymbol{F})+1 .
$$

This type of control resembles the turgor-driven movements of plants, characterised by efficiency and low power consumption. Based on this nature-inspired means of motion, research labs are in the process of developing new and biomimetic actuators [19] (see Figure $2_{a}$ ).

It is shown next that the density $W_{\text {tot }}$, as given by (2.8), enjoys the same polyconvex and coerciveness properties as the stored mechanical energy density $W$.

Proposition 2.1. For each fixed $m \in L^{2}\left(\mathcal{D}_{0}\right)$ and $\omega \in \Omega$, the total stored energy function (2.8) for the turgor pressure control is polyconvex and coercive.

Proof. The turgor-pressure contribution $\hat{W}_{m}$ (2.9) is linear with respect to the Jacobian $J$. Therefore, provided that $W(\omega, \boldsymbol{F})$ is polyconvex, then $W_{\text {tot }}$ is polyconvex. With regards to coerciveness, from (2.4) it follows that

$$
\begin{aligned}
W_{\mathrm{tot}}(\omega, \boldsymbol{F}, m) & \geq c_{0}(\omega)\left(\|\boldsymbol{F}\|^{2}+\|\boldsymbol{H}\|^{2}+J^{2}\right)+c_{1}(\omega)-m(\boldsymbol{X})(J-1) \\
& \geq \frac{c_{0}(\omega)}{2}\left(\|\boldsymbol{F}\|^{2}+\|\boldsymbol{H}\|^{2}+J^{2}\right)+c_{1}(\omega)+\frac{c_{0}(\omega)}{2} J^{2}-m(\boldsymbol{X})(J-1) .
\end{aligned}
$$

The last two last terms in 2.10 are conveniently manipulated as

$$
\frac{c_{0}}{2} J^{2}-m(\boldsymbol{X})(J-1)=\frac{c_{0}}{2}\left[J-\frac{m(\boldsymbol{X})}{c_{0}}\right]^{2}+m(\boldsymbol{X})-\frac{m(\boldsymbol{X})^{2}}{2 c_{0}} \geq m(\boldsymbol{X})-\frac{m(\boldsymbol{X})^{2}}{2 c_{0}},
$$

which permits to conclude that

$W_{\text {tot }}(\omega, \boldsymbol{F}, m) \geq \frac{c_{0}(\omega)}{2}\left(\|\boldsymbol{F}\|^{2}+\|\boldsymbol{H}\|^{2}+J^{2}\right)+\widetilde{c}_{1}(\boldsymbol{X}, \omega) ; \quad \widetilde{c}_{1}(\boldsymbol{X}, \omega)=c_{1}(\omega)+m(\boldsymbol{X})-\frac{m(\boldsymbol{X})^{2}}{2 c_{0}(\omega)}$,

and so $W_{\text {tot }}$ is coercive. 


\subsubsection{Tension fiber type control}

In this case, the control exerts an inner fiber tension $m(\boldsymbol{X}): \mathcal{D}_{0} \rightarrow \mathbb{R}$ along a unitary direction $\boldsymbol{a}(\boldsymbol{X}): \mathcal{D}_{0} \rightarrow \mathbb{R}^{N}$, with $\|\boldsymbol{a}(\boldsymbol{X})\|=1$, in the reference configuration. The energy $\hat{W}_{m}$ is then given by

$$
\hat{W}_{m}(\boldsymbol{F})=-\frac{1}{2}\|\boldsymbol{F} \boldsymbol{a}\|^{2} .
$$

There are relevant examples in nature where this type of actuation is harnessed as a means to achieve a desired motion, such as the complex electro-activation of the human heart, mathematically modelled as in 2.12). In that context, $m$ represents the active cardiomyocyte contraction stress responsible for the deformation of the heart tissue (see Figure 2 , and [20, 21]).

Proposition 2.2. Let $\mu_{1}(\omega)>0$ be the first parameter in the stochastic version of the MooneyRivlin model (2.2). Then, for each $m \in L^{2}\left(\mathcal{D}_{0}\right)$ and $\omega \in \Omega$, with

$$
\sup _{\boldsymbol{X} \in \mathcal{D}_{0}} m(\boldsymbol{X})<2 \mu_{1}(\omega)
$$

the total stored energy density function $W_{\text {tot }}$ in (2.8), with $\hat{W}_{m}$ given by (2.12), is polyconvex and coercive.

Proof. The energy density $W_{\text {tot }}$ can be re-arranged as

$$
\begin{aligned}
W_{\mathrm{tot}}(\omega, \boldsymbol{F}, m) & =\psi_{1}(\omega, \boldsymbol{F})+\psi_{2}(\omega, \boldsymbol{H}, J) \\
\psi_{1}(\omega, \boldsymbol{F}) & =\mu_{1}(\omega)\|\boldsymbol{F}\|^{2}-\frac{m}{2}\|\boldsymbol{F} \boldsymbol{a}\|^{2} ; \\
\psi_{2}(\omega, \boldsymbol{H}, J) & =\mu_{2}(\omega)\|\boldsymbol{H}\|^{2}-2\left(\mu_{1}(\omega)+2 \mu_{2}(\omega)\right) \log J+\frac{\mu_{3}}{2}(J-1)^{2} .
\end{aligned}
$$

Clearly, the function $\psi_{2}(\omega, \boldsymbol{H}, J)$ is convex with respect to $\boldsymbol{H}$ and $J$. Therefore, polyconvexity of $W_{\text {tot }}$ requires convexity of $\psi_{1}(\omega, \boldsymbol{F})$ with respect to $\boldsymbol{F}$. Let us define, for $t \in \mathbb{R}$ and $\boldsymbol{F}_{0}, \boldsymbol{F} \in \mathbb{R}^{N \times N}$, the section

$$
g(\omega, t)=\psi_{1}\left(\omega, \boldsymbol{F}_{0}+t \boldsymbol{F}\right)=\mu_{1}(\omega)\left\|\boldsymbol{F}_{0}+t \boldsymbol{F}\right\|^{2}-\frac{1}{2} m(\boldsymbol{X})\left\|\left(\boldsymbol{F}_{0}+t \boldsymbol{F}\right) \boldsymbol{a}(\boldsymbol{X})\right\|^{2}
$$

The second derivative of $g$ with respect to $t$ yields

$$
\frac{\partial^{2} g}{\partial t^{2}}(\omega, t)=2 \mu_{1}(\omega)\|\boldsymbol{F}\|^{2}-m(\boldsymbol{X})\|\boldsymbol{F} \boldsymbol{a}(\boldsymbol{X})\|^{2} \geq\left(2 \mu_{1}(\omega)-m(\boldsymbol{X})\right)\|\boldsymbol{F}\|^{2} .
$$

It is therefore bounded from below provided that $2 \mu_{1}(\omega)-m(\boldsymbol{X}) \geq K>0$. As a result, $\psi_{1}(\omega, \boldsymbol{F})$ is convex with respect to $\boldsymbol{F}$ and the total contribution $W_{\text {tot }}$, polyconvex. Coerciveness of the total contribution $W_{\text {tot }}$ is straightforward. 


\subsection{Stochastic state laws under the action of bio-inspired controls}

Sections 2.3.1 and 2.3.2 have illustrated under what assumptions, stored energy functions of the type (2.8) with $\hat{W}_{m}$, as those in (2.9) and (2.12), respectively, are polyconvex and coercive.

As shown by Ball in his seminal paper [10], this entails the existence of minimisers $\boldsymbol{\Phi}(\boldsymbol{X}, \omega)$ for each $m \in L^{2}\left(\mathcal{D}_{0}\right)$ and $\omega \in \Omega$ for the total energy functional

$$
\Pi(\omega, \boldsymbol{\Phi}, m):=\int_{\mathcal{D}_{0}} W_{\text {tot }}(\omega, \boldsymbol{F}, m) d \boldsymbol{X},
$$

over the class of mapping fields

$$
\begin{aligned}
\mathbb{U} & :=\left\{\boldsymbol{\Phi}(\omega) \in \mathbb{V}_{D}: \boldsymbol{H}(\omega) \in L^{2}\left(\mathcal{D}_{0} ; \mathbb{R}^{N \times N}\right), \quad J(\omega) \in L^{2}\left(\mathcal{D}_{0}\right), \text { and } J(\omega)>0 \text { a.e. in } \mathcal{D}_{0}\right\} \\
\mathbb{V}_{D} & :=\left\{\boldsymbol{\Phi} \in H^{1}\left(\mathcal{D}_{0} ; \mathbb{R}^{N}\right): \boldsymbol{\Phi}=\boldsymbol{\Phi}_{0} \text { on } \Gamma_{0_{D}}\right\}
\end{aligned}
$$

Remark 1. An additional term of the form

$$
\Pi_{e x t}(\boldsymbol{\Phi}(\boldsymbol{X}, \omega))=-\int_{\mathcal{D}_{0}} \boldsymbol{f}_{0}(\boldsymbol{X}, \omega) \cdot \boldsymbol{\Phi}(\boldsymbol{X}, \omega) d \boldsymbol{X}-\int_{\Gamma_{0_{N}}} \boldsymbol{t}_{0}(\boldsymbol{X}, \omega) \cdot \boldsymbol{\Phi}(\boldsymbol{X}, \omega) d S,
$$

collecting the energy exerted by random, conservative volumetric $\boldsymbol{f}_{0}$ and surface $\boldsymbol{t}_{0}$ loads may be added to (2.16). For the sake of simplicity we do not consider such a term in what follows.

The stationary point $\boldsymbol{\Phi}$ of the functional $\Pi$ in $(2.16)$ solves

$$
\frac{\partial \Pi}{\partial \boldsymbol{\Phi}}(\boldsymbol{\Phi}(\boldsymbol{X}, \omega), m)(\boldsymbol{v})=\int_{\mathcal{D}_{0}} \boldsymbol{P}(\boldsymbol{X}, \omega): \nabla_{0} \boldsymbol{v} d \boldsymbol{X}=0 ; \quad \boldsymbol{v} \in H_{0}^{1}\left(\mathcal{D}_{0} ; \mathbb{R}^{N}\right) ;
$$

$\boldsymbol{P}(\boldsymbol{X}, \omega): \mathcal{D}_{0} \times \Omega \rightarrow \mathbb{R}^{N \times N}$ being the random first Piola-Kirchhoff stress tensor, defined as

$$
\boldsymbol{P}(\boldsymbol{X}, \omega)=\nabla_{\boldsymbol{F}} W_{\text {tot }}(\omega, \boldsymbol{F}, m)=\nabla_{\boldsymbol{F}} W(\omega, \boldsymbol{F})+\nabla_{\boldsymbol{F}} W_{m}(\boldsymbol{F}, m),
$$

where $\boldsymbol{\nabla}_{\boldsymbol{F}} W_{m}(\boldsymbol{F}, m)$ adopts the following expression for the Turgor-pressure and the tension fiber controls in 2.9 and 2.12 , respectively,

$$
\boldsymbol{\nabla}_{\boldsymbol{F}} W_{m}(\boldsymbol{F}, m)=-m \boldsymbol{H} ; \quad \boldsymbol{\nabla}_{\boldsymbol{F}} W_{m}(\boldsymbol{F}, m)=-m(\boldsymbol{F} \boldsymbol{a} \otimes \boldsymbol{a}) .
$$

In equation 2.21), $(\boldsymbol{a} \otimes \boldsymbol{b})_{i j}=a_{i} b_{j}, \forall \boldsymbol{a}, \boldsymbol{b} \in \mathbb{R}^{N}$. Finally, integration by parts in 2.19) enables to obtain the strong or local form of the conservation of the linear momentum, i.e.,

$$
\begin{aligned}
\operatorname{DIV}(\boldsymbol{P}(\boldsymbol{X}, \omega)) & =0 ; & & \text { in } \Omega_{0} \\
(\boldsymbol{P}(\boldsymbol{X}, \omega)) \boldsymbol{N} & =0 ; & & \text { on } \Gamma_{0_{N}} \\
\boldsymbol{\Phi} & =\boldsymbol{\Phi}_{0} ; & & \text { on } \Gamma_{0_{D}}
\end{aligned}
$$

where $\operatorname{DIV}(\bullet)$ represents the material divergence operator, and $\boldsymbol{N}$, the outward vector to the undeformed boundary $\Gamma_{0}$. 
Here is the issue of the potential non-uniqueness of solutions for $(2.22)$. It is true that every minimizer in 2.16) is a solution of (2.22), but neither uniqueness of minimizers of (2.16) is guaranteed nor possible solutions of $(2.22)$ that are not minimizers of (2.16). From an analytical point of view, we resolve this issue by considering, for each feasible $m$, all possible minimizers of (2.16). However, from the point of view of the numerical approximation, one has to work with (2.22), and non-uniqueness can be a real difficulty. It is nonetheless true that our numerical simulations of Section 4.2 have not shown any particular difficulty in this regard.

\section{The control problem under uncertainty}

The objective of the optimal control problem considered in this paper is two-fold: (a) to find a distribution of the control $m(\boldsymbol{X})$ (Turgor pressure or fiber tension) that exerts a deformation on the undeformed domain $\mathcal{D}_{0}$, modifying its shape and deforming it into a desired target deformation $\boldsymbol{\Phi}_{d}$; (b) to minimise the effect of the uncertainty of the material parameters $\left\{\mu_{i}(\omega), 1 \leq i \leq 3\right\}$, for the Mooney-Rivlin model 2.2 in the attainability of the target deformation.

\subsection{Formulation of the optimal control problem}

In this paper, we are mainly interested in the numerical approximation of robust optimal control problems for state laws of the form (2.22). Thus, we introduce a discrete setting and so we assume that uncertainty in the material properties $\left\{\mu_{1}, \mu_{2}, \mu_{3}\right\}$ is quantified by means of a multivariate and discrete random variable $\boldsymbol{Z}$, with scenarios $\boldsymbol{z}^{i}=\left(z_{1}^{i}, z_{2}^{i}, z_{3}^{i}\right) \in \mathcal{R}:=\mathbb{R}_{+}^{3}$ and probabilities $\pi_{i}, 1 \leq i \leq \mathcal{I}$. Continuous distributions may be recovered in the limit $\mathcal{I} \rightarrow \infty$ (see, e.g. [22]).

The random variable $\boldsymbol{Z}$ induces a probability distribution $\mathbb{P}_{\boldsymbol{Z}}$ of the form

$$
\mathbb{P}_{\boldsymbol{Z}}=\sum_{i=1}^{\mathcal{I}} \pi_{i} \delta_{\boldsymbol{z}^{i}}, \quad 0<\pi_{i} \leq 1, \quad \sum_{i=1}^{\mathcal{I}} \pi_{i}=1
$$

where $\delta_{\boldsymbol{z}^{i}}$ is a Dirac mass located at $\boldsymbol{z}^{i}$.

In the context of robust optimal control problems with continuous random variables and/or random fields, a similar discretisation process as the one proposed hereafter takes place (see [18, Chap. 4]). In this case, the robustness of the optimal control is achieved by incorporating a robustness criterion in the functional cost which typically involves the first two statistical moments of the performance. These statistical moments often require the numerical computation of multi-dimensional integrals that cannot be evaluated analytically. This issue has motivated the development of efficient methods to address the problem (such as dimension reduction

methods and sparse grid collocation methods) which discretize the continuous random domain in a finite set of stochastic nodes $\boldsymbol{z}^{i}$ and an associated weights $\pi_{i}$.

For each scenario $\boldsymbol{z}^{i} \in \mathcal{R}, 1 \leq i \leq \mathcal{I}$, we denote by $W^{i}(\mathbf{F})$ the stored energy density

$$
W^{i}(\boldsymbol{F})=z_{1}^{i}\left(\|\boldsymbol{F}\|^{2}-3\right)+z_{2}^{i}\left(\|\boldsymbol{H}\|^{2}-3\right)-2\left(z_{1}^{i}+2 z_{2}^{i}(\omega)\right) \log J+\frac{z_{3}^{i}}{2}(J-1)^{2},
$$


the total energy density $W_{\text {tot }}^{i}(\boldsymbol{F}, m)=W^{i}(\boldsymbol{F})+W_{m}(\boldsymbol{X}, \boldsymbol{F})$, with $W_{m}(\boldsymbol{X}, \boldsymbol{F})$ given by $(2.8)$, and finally the total energy functional

$$
\Pi^{i}(\boldsymbol{\Phi}, m):=\int_{\mathcal{D}_{0}} W_{\text {tot }}^{i}(\boldsymbol{F}, m) d \boldsymbol{X}, \quad \boldsymbol{\Phi} \in \mathbb{U} .
$$

Let $\Gamma_{0}$ be a (measurable) part of the free boundary $\Gamma_{0_{N}}$ in the undeformed configuration $\mathcal{D}_{0}$ and let $\boldsymbol{\Phi}_{d}: \Gamma_{0} \rightarrow \mathbb{R}^{N}$ be a desired deformation. Then, for $m \in H^{1}\left(\mathcal{D}_{0}\right)$, the following tracking-type in surface deformations functional is considered:

$$
\mathcal{J}(m):=\sum_{i=1}^{\mathcal{I}} \pi_{i} \int_{\Gamma_{0}}\left\|\Phi^{i}(\boldsymbol{X})-\boldsymbol{\Phi}_{d}(\boldsymbol{X})\right\|^{2} d S(\boldsymbol{X})+\frac{M}{2} \int_{\mathcal{D}_{0}} m(\boldsymbol{X})^{2} d \boldsymbol{X}+\frac{\epsilon}{2} \int_{\mathcal{D}_{0}}|\nabla m(\boldsymbol{X})|^{2} d \boldsymbol{X},
$$

where $\boldsymbol{\Phi}^{i}$ is a minimizer of 3.2 . Eventually, the robust optimal control problem reads as:

$$
\left\{\begin{array}{l}
\text { Minimize in } m \in H^{1}\left(\mathcal{D}_{0}\right): \quad \mathcal{J}(m) \\
\text { subject to }
\end{array} \quad \boldsymbol{\Phi}^{i} \text { is a minimizer of } 3.2, \quad 1 \leq i \leq \mathcal{I} .\right.
$$

\subsection{Existence of optimal controls}

The goal of this section is to prove the following existence result:

TheOREM 3.1. There is at least an optimal control $m(\boldsymbol{X})$ for problem (3.4).

For the sake of clarity, this will be carried out in the deterministic case and later extended to the stochastic setting.

\subsubsection{Deterministic case}

Throughout this subsection it is assumed that $\mathcal{I}=1$.

Proof. We carefully describe all the steps necessary to prove Theorem 3.1 .

(i) Let $\left\{m_{j}\right\}$ be a minimizing sequence for the control problem (3.4), and let $\left\{\boldsymbol{\Phi}_{j}(\boldsymbol{X})\right\}$ $\left(\boldsymbol{\Phi}_{j} \in \mathbb{U}\right)$ be their corresponding mapping fields which are minimizers of the total energy functional (3.2), which throughout this subsection is denoted by $\Pi$ (recall that $\mathcal{I}=1$ ). Let $\boldsymbol{F}_{j}:=\boldsymbol{\nabla}_{0} \boldsymbol{\Phi}_{j}$, and $\boldsymbol{H}_{j}$ and $J_{j}$ be the co-factor and Jacobian of $\boldsymbol{F}_{j}$, respectively. The regularising part of the objective function $\mathcal{J}$ entails uniform boundedness of $\left\{m_{j}\right\}$ in $H^{1}\left(\mathcal{D}_{0}\right)$. Hence, for a non-relabelled subsequence, $m_{j} \rightarrow m$ in $H^{1}\left(\mathcal{D}_{0}\right)$ and $m_{j} \rightarrow m$ in $L^{2}\left(\mathcal{D}_{0}\right)$, for some feasible $m$.

(ii) Take any feasible $\hat{\boldsymbol{\Phi}} \in \mathbb{U}$ and let $\hat{\boldsymbol{F}}:=\nabla_{0} \hat{\boldsymbol{\Phi}}$. Since $\boldsymbol{\Phi}_{j}$ is a minimiser of $\Pi\left(m_{j}\right)$, then

$$
\Pi\left(\boldsymbol{\Phi}_{j}, m_{j}\right) \leq \Pi\left(\hat{\mathbf{\Phi}}, m_{j}\right) .
$$

The uniform boundedness of $\left\{m_{j}\right\}$ in $L^{2}\left(\mathcal{D}_{0}\right)$ implies the existence of a constant $D$ independent of $j$ which bounds the right-hand side of (3.5). Coerciveness of $W_{\text {tot }}$ then 
implies that $\boldsymbol{\Phi}_{j}$ is uniformly bounded in $H^{1}\left(\mathcal{D}_{0}\right)$. Hence, by [11, Th. 7.6-1], there exists $\Phi \in H^{1}\left(\mathcal{D}_{0} ; \mathbb{R}^{N}\right)$, such that for a not relabelled subsequence,

$$
\boldsymbol{F}_{j} \rightarrow \boldsymbol{F}, \quad \boldsymbol{H}_{j} \rightarrow \boldsymbol{H}, \quad J_{j} \rightarrow J \text { in } L^{2}\left(\mathcal{D}_{0}\right),
$$

where $\boldsymbol{F}:=\boldsymbol{F}\left(\boldsymbol{\nabla}_{0} \boldsymbol{\Phi}\right), \boldsymbol{H}:=\boldsymbol{H}\left(\boldsymbol{\nabla}_{0} \boldsymbol{\Phi}\right), J:=J\left(\boldsymbol{\nabla}_{0} \boldsymbol{\Phi}\right)$.

(iii) Inequality (3.5) may be rewritten as

$$
\Pi\left(\boldsymbol{\Phi}_{j}, m\right)+\int_{\mathcal{D}_{0}}\left(m_{j}-m\right) \hat{W}\left(\boldsymbol{F}_{j}\right) d \boldsymbol{X} \leq \Pi(\hat{\boldsymbol{\Phi}}, m)+\int_{\mathcal{D}_{0}}\left(m_{j}-m\right) \hat{W}(\hat{\boldsymbol{F}}) d \boldsymbol{X} .
$$

Using the strong convergence $m_{j} \rightarrow m$ in $L^{2}\left(\mathcal{D}_{0}\right)$, the weak convergences (3.6), the polyconvexity of $W_{\text {tot }}$ and passing to the limit in (3.7) we conclude that

$$
\Pi(\boldsymbol{\Phi}, m) \leq \Pi(\hat{\mathbf{\Phi}}, m)
$$

The arbitrariness of $\hat{\boldsymbol{\Phi}}$ implies that $\boldsymbol{\Phi}$ is a minimiser of $\Pi(m)$. By using Mazur's theorem, the compactness of the trace operator and following the same lines as in the proof of [11, Th. 4.10-2], one proves that $J>0$ a.e. in $\mathcal{D}_{0}$ and that $\boldsymbol{\Phi}=\boldsymbol{\Phi}_{0}$ on $\Gamma_{0_{D}}$. Therefore, $\boldsymbol{\Phi} \in \mathbb{U}$ is a minimiser of $\Pi$ for the limit control $m$.

(iv) Finally, the weakly lower semicontinuity of the three contributions in the cost functional (3.3) implies that $m$ is indeed a minimiser of problem (3.4).

Remark 2. Notice that the term involving the gradient of the control $m(\boldsymbol{X})$ in $\mathcal{J}$ ensures the strong convergence $m_{j} \rightarrow m$, which is of crucial importance in the previous proof. The same conclusion may be obtained if lower and upper point-wise constraints on $m$, and boundedness of $\nabla m$ in $L^{2}\left(\mathcal{D}_{0}\right)^{N}$ are imposed.

\subsubsection{Stochastic case}

Proof. The proof follows along the same lines as in the deterministic case, given that we only have a finite number of possible scenarios. We highlight the specific ingredients brought by the probabilistic framework.

Let $\left\{m_{j}(\boldsymbol{X})\right\}$ be minimizing for problem (3.4) and let $\left\{\boldsymbol{\Phi}_{j}^{i}(\boldsymbol{X}\}, 1 \leq i \leq \mathcal{I}\right.$, be their corresponding mapping fields which are minimizers of the functional $\Pi^{i}$ in 3.2 for $m=m_{j}$. Similarly to the deterministic case, $m_{j} \rightarrow m$ in $H^{1}\left(\mathcal{D}_{0}\right)$ and $m_{j} \rightarrow m$ in $L^{2}\left(\mathcal{D}_{0}\right)$, for some $m$.

From the proof of the deterministic case, it follows that for each $1 \leq i \leq \mathcal{I}$, there exists $\boldsymbol{\Phi}^{i}$, weak limit in $\mathbb{V}_{D}$ of (a subsequence of) $\boldsymbol{\Phi}_{j}^{i}$, that minimizes $\Pi^{i}$ in 3.2 for the limit control $m(\boldsymbol{X})$. Since there is only a finite number of possibilities $1 \leq i \leq \mathcal{I}$, we can select a subsequence $j$, not relabelled, which is valid for all such $i$ simultaneously, and

$$
m_{j} \rightarrow m, \quad \boldsymbol{\Phi}_{j}^{i} \rightarrow \boldsymbol{\Phi}^{i}, \quad \text { in } H^{1}\left(\mathcal{D}_{0}\right)
$$

for all $i$. The result then follows taking into account that all the terms involved in the cost functional $\mathcal{J}(m)$, as given by $(3.3)$, are weakly lower semicontinuous. 


\section{Numerical resolution method and numerical experiments}

\subsection{Gradient-based optimisation algorithm}

In this paper, we advocate for a gradient-based optimisation method for the numerical approximation of the robust optimal control problem (3.4). As customary in this type of methods, in order to compute a descent direction, we use the standard Lagrangian method. To this end, let us consider the Lagrangian $\mathcal{L}$ defined as

$$
\begin{aligned}
\mathcal{L}(\overline{\boldsymbol{\Phi}}, \overline{\boldsymbol{p}}, \bar{m})= & \sum_{i=1}^{\mathcal{I}} \pi_{i} \int_{\Gamma_{0}}\left\|\overline{\boldsymbol{\Phi}}^{i}(\boldsymbol{X})-\boldsymbol{\Phi}_{d}(\boldsymbol{X})\right\|^{2} d S(\boldsymbol{X})+\frac{M}{2} \int_{\mathcal{D}_{0}} \bar{m}(\boldsymbol{X})^{2} d \boldsymbol{X}+\frac{\epsilon}{2} \int_{\mathcal{D}_{0}}|\nabla \bar{m}(\boldsymbol{X})|^{2} d \boldsymbol{X} \\
& -\sum_{i=1}^{\mathcal{I}} \int_{\mathcal{D}_{0}} \overline{\boldsymbol{P}}^{i}: \nabla_{0} \overline{\boldsymbol{p}}^{i} d \boldsymbol{X}
\end{aligned}
$$

where $\overline{\boldsymbol{\Phi}}=\left(\bar{\Phi}^{1}, \cdots, \bar{\Phi}^{\mathcal{I}}\right), \overline{\boldsymbol{p}}=\left(\overline{\boldsymbol{p}}^{1}, \cdots, \overline{\boldsymbol{p}}^{\mathcal{I}}\right) \in\left(H^{1}\left(\mathcal{D}_{0} ; \mathbb{R}^{N}\right)\right)^{\mathcal{I}}$, and $\overline{\boldsymbol{P}}^{i}$ is the first PiolaKirchhoff stress tensors associated with scenario $i, 1 \leq i \leq \mathcal{I}$, as given by (2.20) with $\omega$ replaced by $\boldsymbol{z}^{i}$ and $\boldsymbol{F}$ by $\boldsymbol{F}^{i}=\nabla_{0} \overline{\boldsymbol{\Phi}}^{i}$.

Notice that in 4.1$),(\overline{\boldsymbol{\Phi}}, \overline{\boldsymbol{p}}, \bar{m})$ are considered as independent variables.

Let $(\boldsymbol{\Phi}, \boldsymbol{p}, m)$ be a stationary point of $\mathcal{L}$. The stationary condition of $\mathcal{L}$ with respect to $\overline{\boldsymbol{p}}_{i}$ coincides with the stationary condition of the functional $\Pi 2.16$ ) with respect to $\overline{\boldsymbol{\Phi}}$ (see equation (2.19) for each scenario $\boldsymbol{z}^{i}, 1 \leq i \leq \mathcal{I}$, i.e.

$$
\frac{\partial \mathcal{L}}{\partial \overline{\boldsymbol{p}}^{i}}(\boldsymbol{\Phi}, \boldsymbol{p}, m)(\boldsymbol{v})=-\int_{\mathcal{D}_{0}} \boldsymbol{P}^{i}: \boldsymbol{\nabla}_{0} \boldsymbol{v} d \boldsymbol{X}=0, \quad \forall \boldsymbol{v} \in H_{0}^{1}\left(\mathcal{D}_{0}\right)
$$

Equation (4.2) is nonlinear. A consistent linearisation of 4.2 is carried out by means of the standard Newton-Raphson method.

The stationary condition of the Lagrangian $\mathcal{L}$ with respect to $\overline{\boldsymbol{\Phi}}^{i}$ yields

$$
\int_{\mathcal{D}_{0}} \boldsymbol{\nabla}_{0} \boldsymbol{p}^{i}: \mathcal{C}^{i}: \boldsymbol{\nabla}_{0} \boldsymbol{v} d \boldsymbol{X}=\sum_{i=1}^{\mathcal{I}} \pi_{i} \int_{\Gamma_{0}}\left(\boldsymbol{\Phi}^{i}-\boldsymbol{\Phi}_{d}\right) \cdot \boldsymbol{v} d S(\boldsymbol{X}), \quad \forall \boldsymbol{v} \in H_{0}^{1}\left(\mathcal{D}_{0}\right)
$$

$\mathcal{C}^{i}=\mathcal{C}^{i}\left(\boldsymbol{X}, \boldsymbol{z}^{i}, m\right)$ being the fourth order elasticity tensor, defined as

$$
\mathcal{C}^{i}\left(\boldsymbol{X}, \boldsymbol{z}^{i}, m\right)=\nabla_{\boldsymbol{F} \boldsymbol{F}}^{2} W\left(\boldsymbol{z}^{i}, \boldsymbol{F}^{i}\right)+\partial_{\boldsymbol{F} \boldsymbol{F}}^{2} W_{m}\left(\boldsymbol{X}, \boldsymbol{F}^{i}\right)
$$

where the expression for $\partial_{\boldsymbol{F} F}^{2} W_{m}(\boldsymbol{X}, \boldsymbol{F})$ for both the Turgor-pressure and fiber tension control contributions are

$$
\partial_{\boldsymbol{F} \boldsymbol{F}}^{2} W_{m}(\boldsymbol{X}, \boldsymbol{F})=-\frac{m}{J}\left(H_{i I} H_{j J}-H_{i J} H_{j I}\right) ; \quad \partial_{\boldsymbol{F} \boldsymbol{F}}^{2} W_{m}(\boldsymbol{X}, \boldsymbol{F})=-\delta_{i j} m a_{I} a_{J},
$$

respectively, and where $\delta_{i j}$ denotes the $i j$ th component of the Kronecker Delta tensor. 
Finally, the directional derivative of the Lagrangian $\mathcal{L}$ with respect to the control $\bar{m}$ in the direction $\hat{m}$ permits to obtain the descend direction. Precisely,

$$
\begin{aligned}
\frac{\partial \mathcal{L}}{\partial \bar{m}}(\boldsymbol{\Phi}, \boldsymbol{p}, m)(\hat{m}) & =\sum_{i=1}^{\mathcal{I}}\left(\int_{\mathcal{D}_{0}} \frac{\partial\left(\partial_{\boldsymbol{F}} W_{m}\right)\left(\boldsymbol{X}, \boldsymbol{F}^{i}\right)}{\partial \bar{m}}(\hat{m}): \nabla_{0} \boldsymbol{p}^{i} d \boldsymbol{X}\right) \\
& +M \int_{\mathcal{D}_{0}} m \hat{m} d \boldsymbol{X}+\varepsilon \int_{\mathcal{D}_{0}} \boldsymbol{\nabla}_{0} m \cdot \nabla_{0} \hat{m} d \boldsymbol{X}
\end{aligned}
$$

where the expression of $\frac{\partial\left(\partial_{\boldsymbol{F}} W_{m}(\boldsymbol{X}, \boldsymbol{F})\right)}{\partial \bar{m}}(\hat{m})$ for both the Turgor-pressure and fiber tension control contributions in 2.9 and 2.12 are, respectively

$$
\frac{\partial\left(\partial_{\boldsymbol{F}} W_{m}\right)}{\partial \bar{m}}(\hat{m})=-\hat{m} \boldsymbol{H} ; \quad \frac{\partial\left(\partial_{\boldsymbol{F}} W_{m}\right)}{\partial \bar{m}}(\hat{m})=-\hat{m}(\boldsymbol{F} \boldsymbol{a} \otimes \boldsymbol{a}) .
$$

\subsection{Numerical experiments}

To keep track of the evolution of simulations during the optimisation procedure, we have introduced a sequence of discrete optimisation iterations akin to a pseudo-time parameter $\tau=\left\{\tau_{0}, \ldots, \tau_{m}\right\}$. At each discrete pseudo-time $\tau$, the pseudo-time evolving control $m(\boldsymbol{X}, \tau)$ induces a random deformation on the undeformed configuration $\mathcal{D}_{0}$. For a random realization $\boldsymbol{z}^{i}$ of the material parameters, the undeformed configuration is transformed into $\mathcal{D}^{i}(m(\boldsymbol{X}, \tau))$ according to

$$
\mathcal{D}^{i}(m(\boldsymbol{X}, \tau))=\boldsymbol{\Phi}^{i}(m(\boldsymbol{X}, \tau))\left(\mathcal{D}_{0}\right),
$$

where $\boldsymbol{\Phi}^{i}=\boldsymbol{\Phi}^{i}(m)$ is a minimiser of 2.16 associated with $m$, as described earlier (see Figure 3).

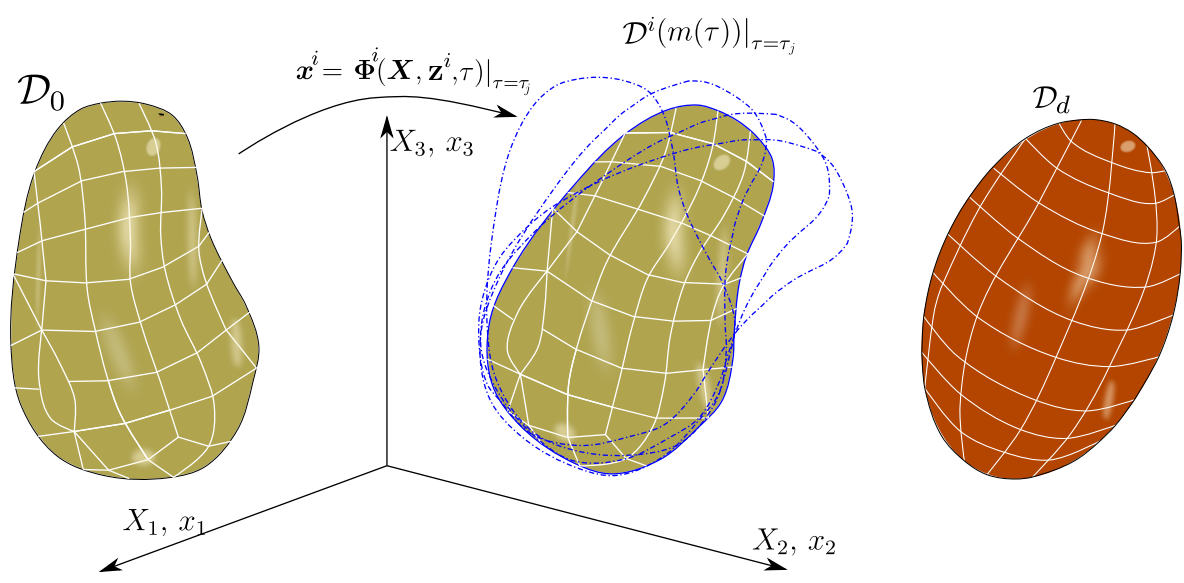

Figure 3: Undeformed configuration $\mathcal{D}_{0}$; Deformed configurations at pseudo-time $\tau_{j}$ for random realizations of $\boldsymbol{z}$ (blue dotted lines), reached after the application of the control $\left.m(\tau)\right|_{\tau=\tau_{j}}$; Target or desired configuration.

In the two numerical experiments that follow, for simplicity, the material parameter $\mu_{2}$ in (2.2) has been taken igual to zero. $\mu_{1}$ and $\mu_{3}$ are modeled as two independent random variables following a Gamma distribution with means 1 and 3, and standard deviations 0.15 and 0.45, 
respectively. The continuous Gamma distributions are discretized by means of an isotropic sparse grid stochastic collocation method [23] leading to a finite set of scenarios $\boldsymbol{z}^{i}$ depicted in Figure 4. Very similar cualitative results are obtained when the number stochastic nodes is increased.

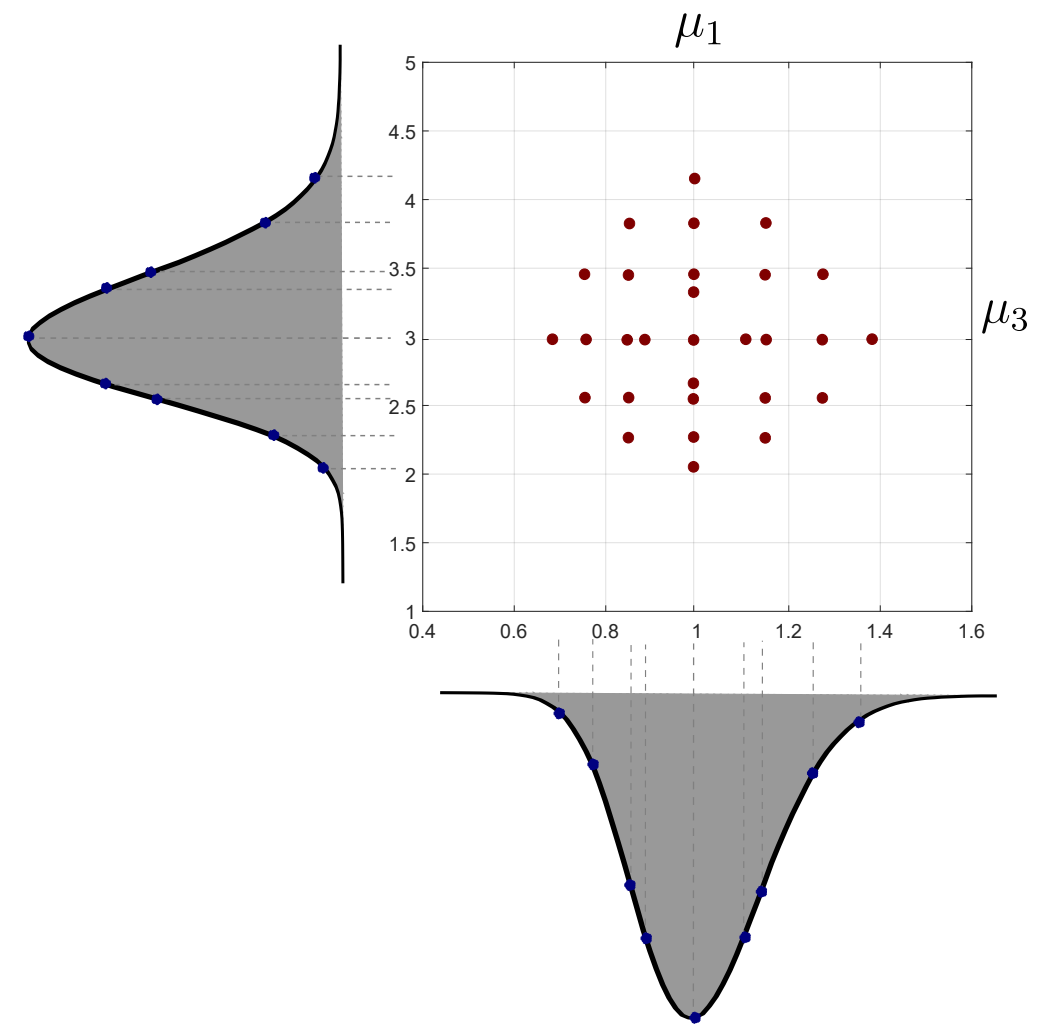

Figure 4: Nodes of the considered isotropic sparse grid for uncertainty quantification in the material parameters $\mu_{1}$ and $\mu_{3}$ in 2.2 .

\subsubsection{Numerical experiment 1: Turgor pressure control}

In this example, a Turgor-pressure type control (see equation (2.9)) is considered. Lower and upper bounds are imposed on the control variable, namely, $-1 \leq m(\boldsymbol{X}) \leq 1$ for all $\boldsymbol{X} \in \mathcal{D}_{0}$. The undeformed configuration $\mathcal{D}_{0}$ is shown in Figure 5. The prismatic-shaped domain $\mathcal{D}_{0}$ has lengths $\left\{L_{X}, L_{y}, L_{z}\right\}=\{10,3,1\}$ parallel to the $\{O X, O Y, O Z\}$ axis. The design domain is tessellated using a $24 \times 4 \times 2$ regular mesh of linear hexahedral elements. $\mathcal{D}_{0}$ is completely fixed in the surface corresponding to the minimum $X$ coordinate. The image of the target configuration $\boldsymbol{\Phi}_{d}$ is also shown in Figure 5 (see black surface labeled as Target Surface (Spatial configuration)). $\Gamma_{0}$ is denoted by Material configuration in Figure 5.

Figure 6 represents the deformed configuration obtained by means of the deterministic control, i.e. taking the material parameters $\mu_{1}=1$ and $\mu_{3}=3$. In this case, the controllable part $\Gamma_{0}$ of the boundary perfectly matches the target configuration (both are overlapped) and 


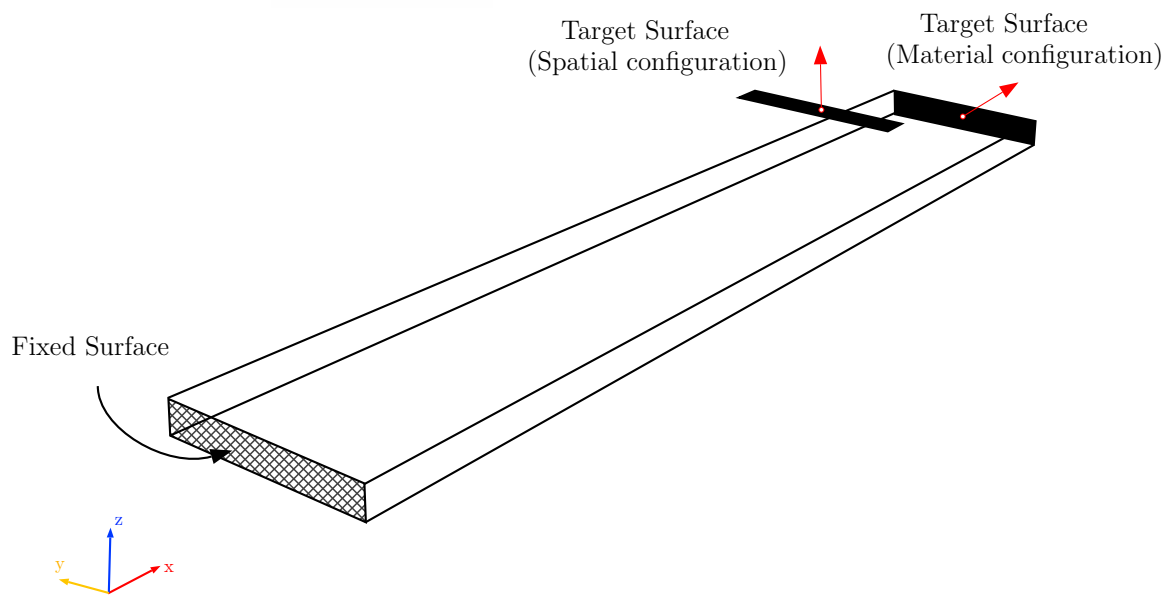

Figure 5: Numerical experiment 1: Problem configuration.

a value of the objective function of $\mathcal{J}=1.28 e-3$ is obtained.

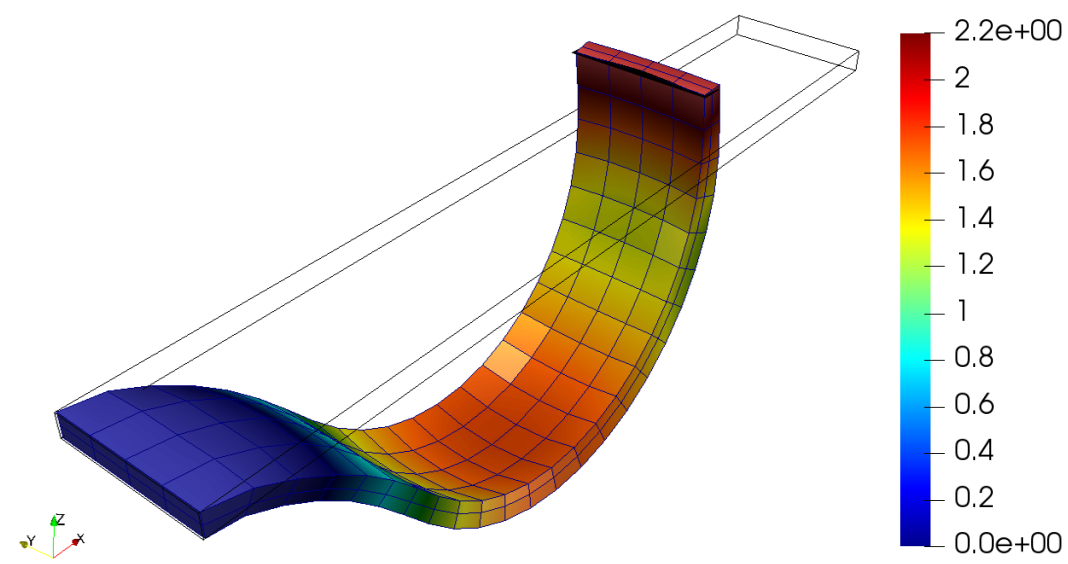

Figure 6: Numerical example 1. Deformed configuration after application of the optuimal control in the deterministic setting. Contour plot distribution of the $L^{2}$ norm of the displacement field $\boldsymbol{u}:=\boldsymbol{x}-\boldsymbol{X}$.

Figures 7 , and 7 , show the mean deformed configuration for the deterministic control after computing all the deformed configurations associated with all the realizations $\boldsymbol{z}^{i}$. The value of the averaged objective function $\mathcal{J}$ in this case is $\mathcal{J}=0.0193$. Clearly, the mean deformed configuration of the $\Gamma_{0}$ is now considerably far from the target configuration. On the contrary, Figures 7 , and $7 t_{t}$ show the mean deformed configuration for the robust control after computing all the deformed configurations associated with all the scenarios $\boldsymbol{z}^{i}$. In this case, the mean deformed configuration of $\Gamma_{0}$ is extremely close to the target (they seem overlapped in the Figure), resulting in a value of the averaged cost functional of $\mathcal{J}=0.0107$. Crucially, this indicates that, as expected, the robust control (which takes into account the stochastic nature 
of the material parameters is less sensitive to dispersion in their random values. This is also corroborated by the lower value of the variance of the $L^{2}$ norm of the displacement field $\boldsymbol{u}:=$ $\boldsymbol{x}-\boldsymbol{X}$ in Figure $7_{l}$ (for robust control) and opposed to Figure 7 , (deterministic control).

Furthermore, Figure 8 shows the contour plot distribution of the control for the deterministic and robust cases from two different geometrical perspectives.

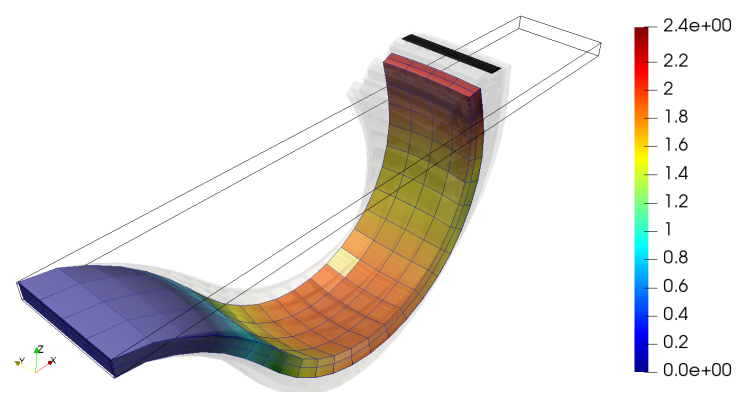

(a)

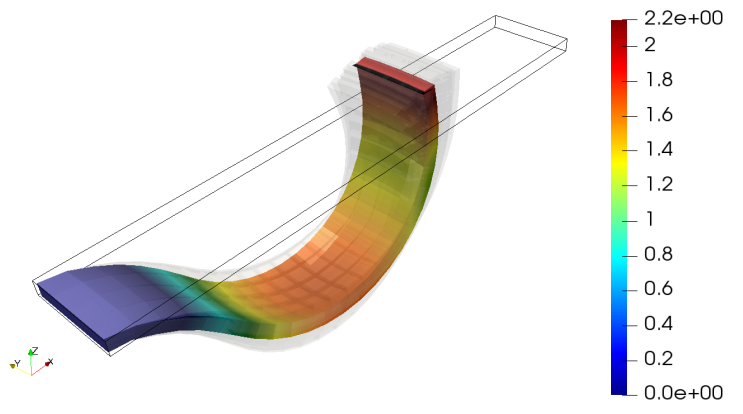

(c)

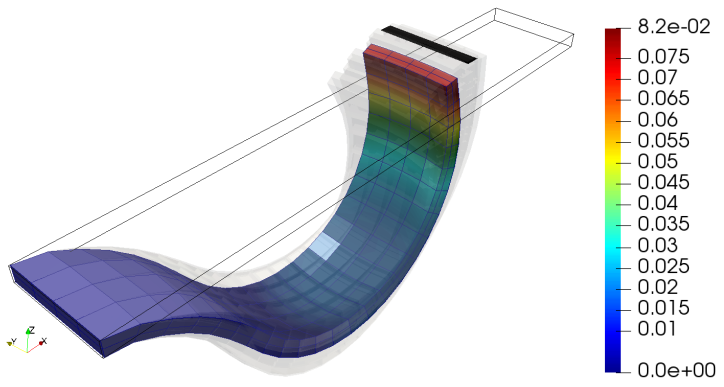

(b)

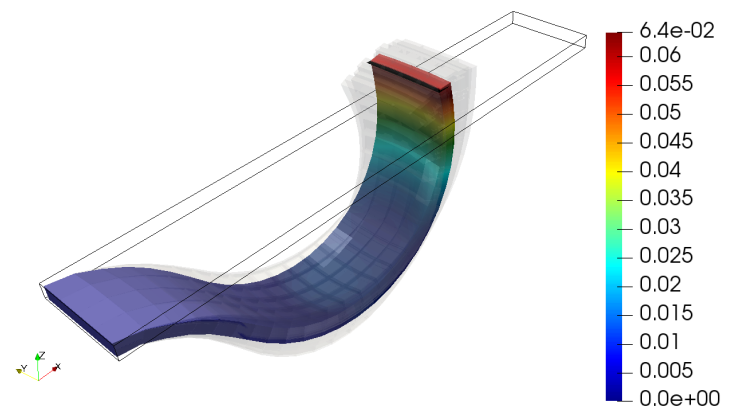

(d)

Figure 7: Numerical example 1. Mean deformed configuration after evaluating all the realizations $\boldsymbol{z}^{i}$ of the material parameters for deterministic control (first row) and robust control (second row). The transparent configurations represent the deformed configurations associated with all the realizations $\boldsymbol{z}^{i}$. Control plot distribution of: mean of the $L^{2}$ norm of $\boldsymbol{u}$ for (a) deterministic and (c) robust control; variance of the $L^{2}$ norm of $\boldsymbol{u}$ for (b) deterministic and (d) robust control.

\subsection{Numerical experiment 2: Active Fibers control}

In this experiment, a fiber tension type control (see equation (2.12)) is considered. Fibers are oriented along the longitudinal direction (see Figure 11). The control variable is constrained according to $0 \leq m(\boldsymbol{X}) \leq 1$. The undeformed configuration $\mathcal{D}_{0}$, shown in Figure 9 , is composed of four prismatic-shaped branches with lengths $\left\{L_{X}, L_{y}, L_{z}\right\}=\{10,3,1\}$ parallel to the $\{O X, O Y, O Z\}$ axis. Similarly to experiment 1, each branch of the domain is tessellated using a $24 \times 4 \times 2$ regular mesh of linear hexahedral elements. The target surface (black surface labeled as Target Surface (Spatial configuration)) and zero Dirichlet boundary conditions (Fixed surface) are shown in Figure 9.

Figures 10, and 10, show the mean deformed configuration for the deterministic control after computing all the deformed configurations associated with all the realizations $\boldsymbol{z}^{i}$. The 


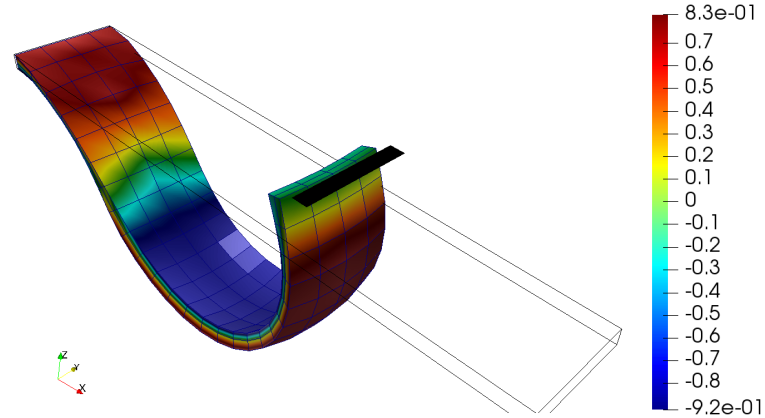

(a)

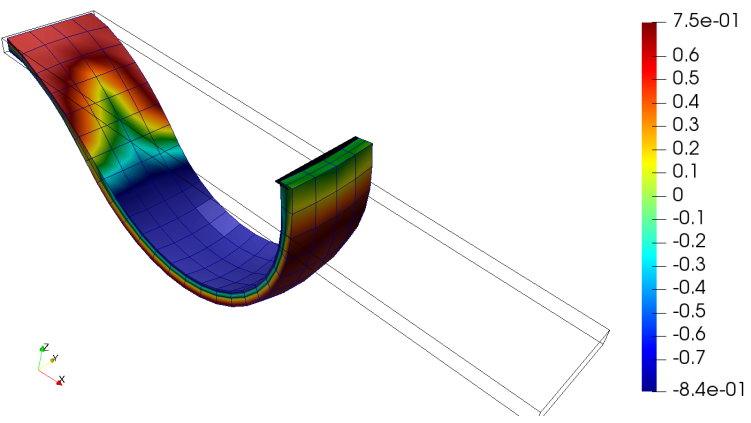

(c)

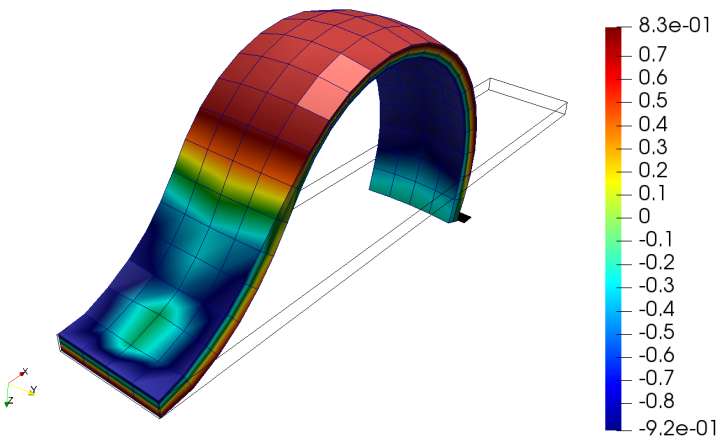

(b)

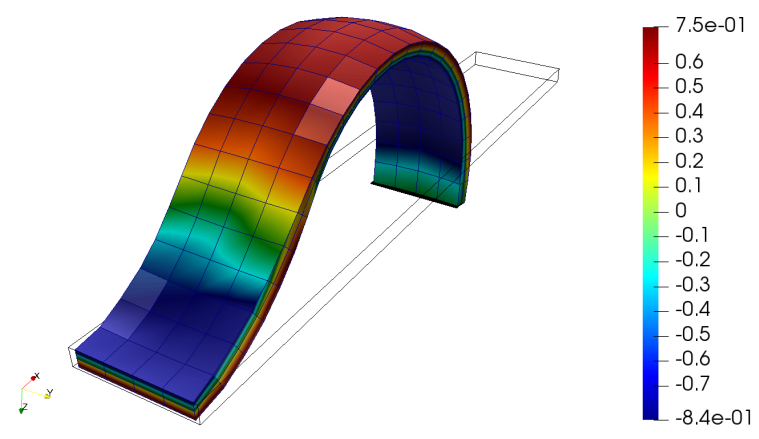

(d)

Figure 8: Numerical example 2. First row: mean deformed configuration for deterministic control; Second row: mean deformed configuration for robust control. Top (a) and bottom (b) view for the deterministic control; Top (c) and bottom (d) view for the robust control.

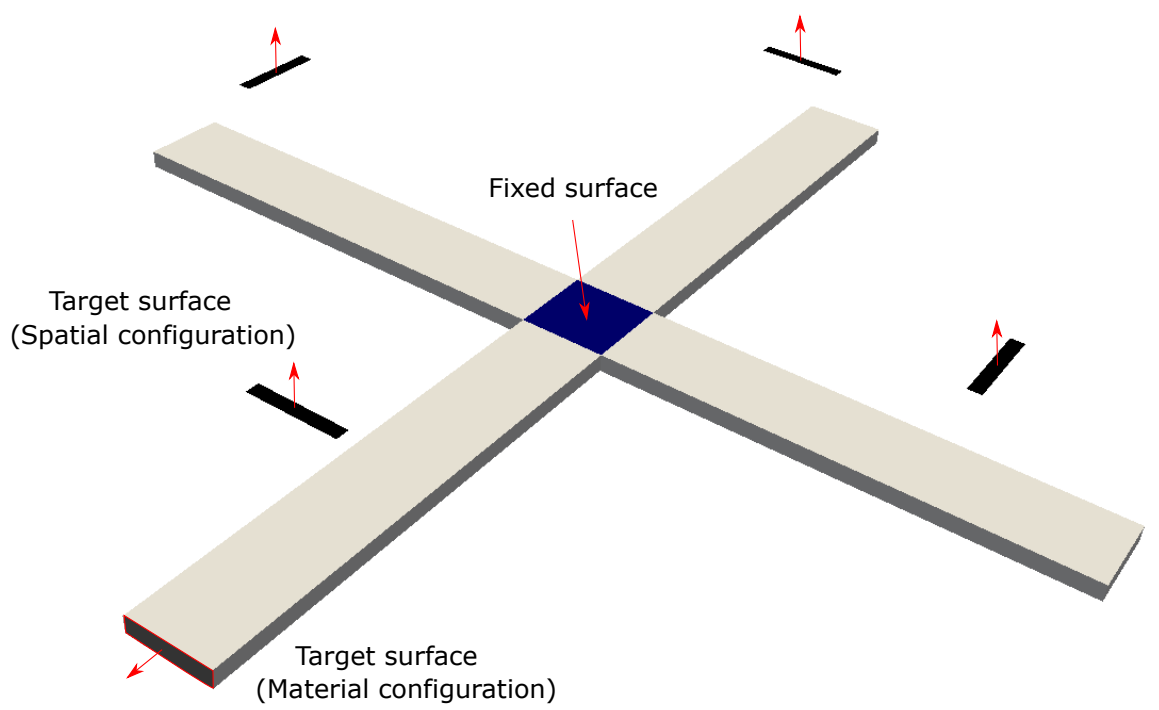

Figure 9: Numerical experiment 2: Problem configuration. 
value of the averaged objective function $\mathcal{J}$ in this case is $\mathcal{J}=0.0140$. Clearly, the mean deformed configuration of the controllable portion $\Gamma_{0}$ is now considerably far from the target configuration. On the contrary, Figures 10, and 10, show the mean deformed configuration for the robust control after computing all the deformed configurations associated with all the realizations $\boldsymbol{z}^{i}$. In this case, the mean deformed configuration of $\Gamma_{0}$ is extremely close to the target, resulting in a value of the averaged cost functional of $\mathcal{J}=0.0074$. As in the previous example, but now with a different type of control, this indicates that the robust control is less sensitive to dispersion in the values of the material parameters. This is also corroborated by the lower value of the variance of the $L^{2}$ norm of the displacement field in Figure 10 (for robust control) and opposed to Figure 10 , (deterministic control).

Finally, Figures 11, and 11, show the mean deformed configuration for the deterministic and robust controls, respectively after computing all the deformed configurations associated with all the realizations $\boldsymbol{z}^{i}$, seeing from a different geometrical perspective as in Figure 10. Notice also that the results have been rotated after applying rotations of $\{\pi / 2, \pi,-\pi / 2\}$ with respect to the $O Z$ axis, resulting in the four-legged actuator shown in Figure 11 . In this Figure, the contour plot distribution of the control (in the deformed configuration, i.e., $m\left(\boldsymbol{\Phi}^{-\mathbf{1}}(\boldsymbol{x})\right)$ ) for both deterministic and robust settings is shown.

\section{Conclusions and perspectives}

This paper is a first step towards the very challenging problem of mathematical analysis and numerical approximation of robust optimal control problems constrained by the system of nonlinear elasticity with uncertainty in the material parameters. Precisely, we have analysed two types of bio-inspired controls: turgor pressure and fiber tension. Although the deterministic situation was numerically explored for the first time in [8], a rigorous mathematical proof of existence of optimal solutions for both problems in the deterministic and stochastic frameworks is presented in this manuscript. Although we have only considered a tracking-type in surface deformations cost functional, the theoretical results and numerical methods here exposed may be extended to other cost functional like the desired direction functional introduced in [8].

The analysis carried out in this paper focuses on the case of discrete distributions for the material parameters. A more sophisticated study is required in order to examine the more challenging cases of continuous distributions and random fields. A number of difficulties appear in the case of continuous randon variables and fields. Among them, the smooth selection of minimisers (in particular, measurability of the equilibrium configurations $\boldsymbol{\Phi}(\boldsymbol{X}, \omega)$ with respect to the random parameter $\omega$, which is required to be able to compute statistical moments of such configurations) and the existence itself of optimal solutions for the underlying optimal control problem.

Also, as is well-known in the case case of linear PDEs [2] convergence of stochastic collocation

methods is a consequence of the regularity of the solution of the underlying PDE with respect to the random paramter. This regularity issue is a very challenging and open problem in the 


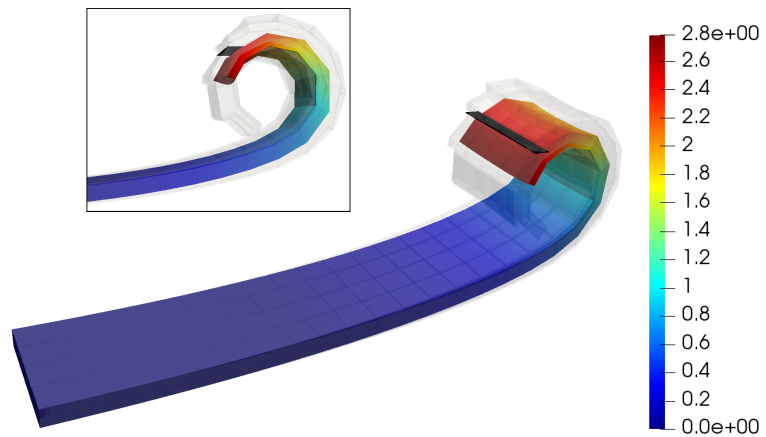

(a)

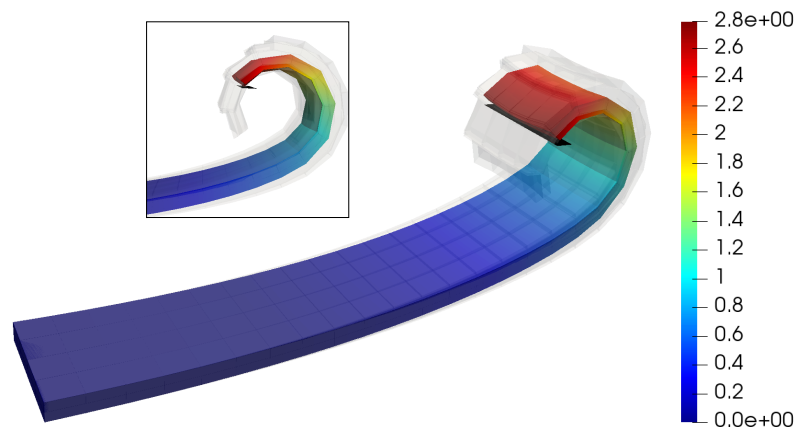

(c)

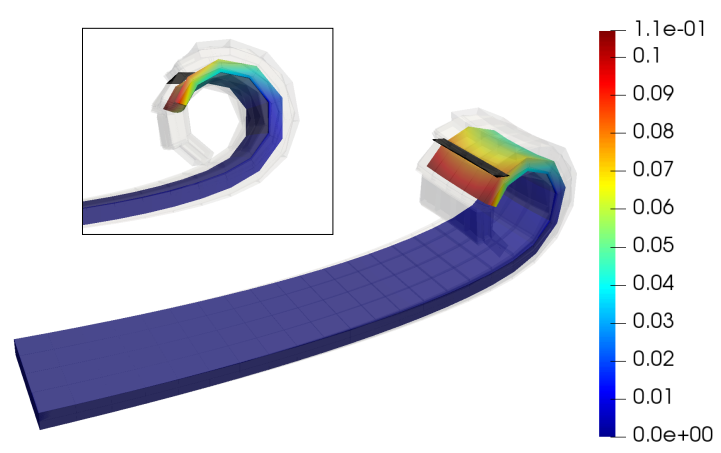

(b)

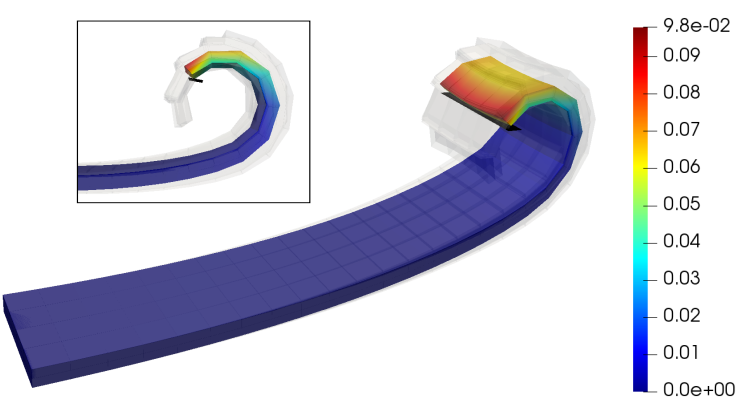

(d)

Figure 10: Numerical example 2. Mean deformed configuration after evaluating all the realizations $\boldsymbol{z}^{i}$ of the material parameters for deterministic control (first row) and robust control (second row). The transparent configurations represent the deformed configurations associated with all the realizations $\boldsymbol{z}^{i}$. Control plot distribution of: mean of the $L^{2}$ norm of $\boldsymbol{u}$ for (a) deterministic and (c) robust control; variance of the $L^{2}$ norm of $\boldsymbol{u}$ for (b) deterministic and (d) robust control.

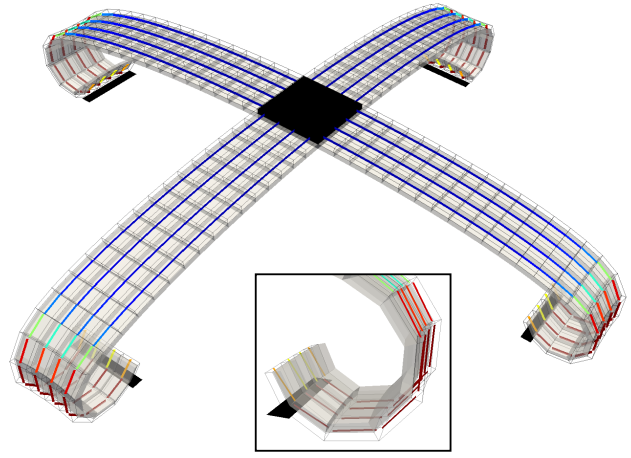

(a)
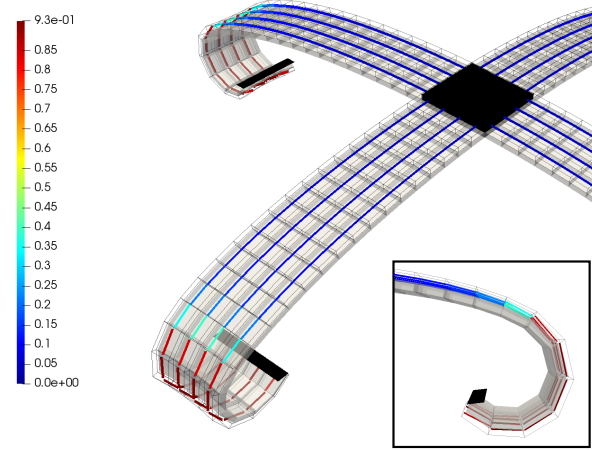

(b)

Figure 11: Numerical example 2. Mean deformed configuration for deterministic control; Second row: mean deformed configuration or robust control. Control plot distribution of the (a) deterministic and (b) robust control along the fibers in the deformed configuration. 
nonlinear situation considered in this paper. Nonetheless, the examples analysed in Subsection 4 show numerical evidence that convergence of stochastic collocation methods holds, as the consideration of finer discrete probability distributions do not introduce numerical instabilities.

Acknowledgements. This research was partially supported by the AEI/FEDER and UE under the contracts DPI2016-77538-R and MTM2017-83740 and by the Fundacion Séneca (Agencia de Ciencia y Tecnología de la Región de Murcia (Spain)) under the contract 20911/PI/18. The second author acknowledges the support provided by Ministerio de Ciencia, Innovación y Universidades, for the award of a Juan de la Cierva Formación Fellowship.

\section{References}

[1] C. Majidi, Soft-matter engineering for soft robotics, Adv. Mater. Technol. 4 (2) (2019) 1800477.

[2] I. Babuska, F. Nobile, R. Tempone, A stochastic collocation method for elliptic partial differential equations with random input data, SIAM J. Numer. Anal. 45 (3) (2007) 10051034.

[3] A. Cohen, R. DeVore, Approximation of high-dimensional parametric pdes, Acta Numer. 24 (2015) 1-159.

[4] M. Gunzburger, C. Webster, G. Zhang, Stochastic finite element methods for partial differential equations with random input data, Acta Numer. 23 (2014) 521-650.

[5] B. Staber, J. Guilleminot, Stochastic modeling of a class of stored energy functions for incompressible hyperelastic materials with uncertainties, C. R. Mecanique 349 (2015) 503514.

[6] B. Staber, J. Guilleminot, Stochastic modeling of the ogden class of stored energy functions for hyperelastic materials: the compressible case, ZAMM Z. Angew. Math. Mech. Engrg. 97 (2017) 273-295.

[7] B. Staber, J. Guilleminot, A random field model for anisotropic strain energy functions and its application for uncertainty quantification in vascular mechanics, Comput. Methods Appl. Mech. Engrg. 333 (2018) 94-113.

[8] A. Günnel, R. Herzog, Optimal control problems in finite strain elasticity by inner pressure and fiber tension, Front. Appl. Math. Stat. 2 (4) (2016).

[9] L. Lubkoll, A. Schuela, M. Weiser, An optimal control problem in polyconvex hyperelasticity, SIAM J. Control Optim. 52 (3) (2014) 1403-1422. 
[10] J. M. Ball, Convexity conditions and existence theorems in nonlinear elasticity, Arch. Rational Mech. Anal. 63 (4) (1977) 337-403.

[11] P. G. Ciarlet, Mathematical elasticity. Vol. I, Vol. 20 of Studies in Mathematics and its Applications, North-Holland Publishing Co., Amsterdam, 1988.

[12] P. Pedregal, Variational methods in nonlinear elasticity, SIAM, Philadelphia, PA, 2000. doi:10.1137/1.9780898719529.

URL http://dx .doi .org/10.1137/1.9780898719529

[13] E. Zuazua, Averaged control, Automatica 50 (2014) 3077-3087.

[14] M. Lazar, E. Zuazua, Averaged control and observation of parameter-depending wave equations, C. R. Mathematiques 352 (6) (2014) 497-502.

[15] Q. Lü, E. Zuazua, Averaged controllability for random evolution partial differential equations, J. Math. Pures Appl. 105 (3) (2016) 367-414.

[16] F. Marín, J. Martínez-Frutos, F. Periago, Robust averaged control of vibrations for the bernoulli-euler beam equation, J. Optim. Theory Appl. 174 (2) (2017) 428-454.

[17] J. Martínez-Frutos, M. Kessler, A. Münch, F. Periago, Robust optimal robin boundary control for the transient heat equation with random input data, Inter. J. Numer. Methods Engrg. 108 (2) (2017) 116-135.

[18] J. Martínez-Frutos, F. Periago, Optimal control of PDEs under uncertainty. An introduction with application to optimal shape design of structures, SpringerBriefs in Mathematics. BCAM SpringerBriefs, Springer, 2018.

[19] I. Must, E. Sinibaldi, B. Mazzolai, A variable-stiffness tendril-like soft robot based on reversible osmotic actuation, Nat. Commun. 10 (2019) 344.

[20] E. García-Blanco, R. Ortigosa, A. Gil, C. H. Lee, J. Bonet, A new computational framework for electro-activation in cardiac mechanics, Comput. Methods Appl. Mech. Engrg. 348 (2019) 796-845.

[21] E. García-Blanco, R. Ortigosa, A. Gil, J. Bonet, Towards an efficient computational strategy for electro-activation in cardiac mechanics, Comput. Methods Appl. Mech. Engrg. 356 (2019) 220-260.

[22] O. Schrempf, D. Brunn, U. Hanebeck, Density approximation based on dirac mixtures with regard to nonlinear estimation and filtering, Proceedings of the 45th IEEE Conference on Decision and Control. San diego, CA, USA (2006).

[23] J. Martínez-Frutos, M. Kessler, F. Periago, Robust optimal shape design for an elliptic pde under uncertainty in its input data, ESAIM: COCV 21 (4) (2015) 901-923. 